\title{
Efficient Cryo-EM: Measuring Effects of Particle Orientation in Electron Microscopy
}

\author{
Katerina Naydenova ${ }^{1,2}$, Christopher J. Russo ${ }^{1}$ \\ 1. MRC Laboratory of Molecular Biology, Cambridge, United Kingdom \\ 2. Trinity College, University of Cambridge, Cambridge, United Kingdom
}

Recent technological advances have ushered in a new era of structure determination by single particle electron cryomicroscopy (cryo-EM) [1]. Still, the specimen and how it interacts with the surface of the support can limit and even prevent structure determination. This phenomenon manifests itself in specific, yet unpredictable particle-surface interactions which limit the number of projection views present in a typical single-particle cryo-EM dataset.

Here we describe a statistical quantity, the efficiency, which characterizes the particle orientation distribution by its ability to provide uniform information and resolution in all directions of the resulting reconstruction. The efficiency is derived from the optical point spread function imposed on the three-dimensional reconstruction by the geometry of the orientation distribution. This, coupled with the assumption that the fall-off in information with resolution can be modelled as a Gaussian function characterized by a $B$-factor, [2], allows us to compute the normalized Fourier-space information transmission that corresponds to a particular orientation distribution.

Efficiency, as defined here, has four useful properties. First, it is linearly related to the amount of solid angle absent in the Fourier density map. Second, it can be determined accurately with only a small number of particles ( 1000). Third, a perfect orientation distribution would have an efficiency equal to 1 , with a value of 0.5 being the approximate threshold between a distribution that will successfully reach high resolution ( $>1 / 4 \AA$ ) and one that will not, using current technology and a typical amount of data ( $\sim 10^{5}$ particle images). Fourth, the efficiency is specifically a property of the orientation distribution, and only weakly depends on other factors that can affect resolution, such as image quality, specimen heterogeneity and data processing algorithms. In this way we isolate an important factor in cryo-EM structure determination, making it amenable to analysis and optimization.

This theoretical framework is implemented in an open source program called cryoEF. It takes the Euler angles assigned to a particle set from any of the popular 3D reconstruction programs and calculates Fourier space information transmission, point spread function, efficiency, directions of highest/lowest resolution, and recommends goniometer tilt angles expected to yield greatest improvement in resolution. We demonstrate this improvement experimentally; tilting the specimen can increase the efficiency in some instances, but it cannot overcome the problems of reduced image contrast and the potentially destructive molecule-surface interactions indicated by an anisotropic orientation distribution [3].

The method described here allows rapid and rigorous evaluation of specimen support materials and experimental conditions during sample preparation. Given a dataset, one can use it to estimate the number of particles required to reach a particular resolution. One can then rationally decide whether it would be better to collect more data or try to improve the orientation distribution through the use of tilt, specimen supports or alternative preparation methods. The method will also facilitate further development of support surfaces like modified graphene, specifically tuned to match the specimen under 
study [4], reduce potentially detrimental interactions and optimize the orientation distribution for structure determination with minimal data.

\section{References:}

[1] J Frank, Nature Protocols 12 (2017), p. 209-212

[2] P B Rosenthal, R Henderson, Journal of Molecular Biology 333 (2003), p. 721-745

[3] R M Glaeser, B-G Han, Biophysics Reports (2016), p. 1-7

[4] C J Russo, L A Passmore, Nature Methods 11 (2014), p. 649-652
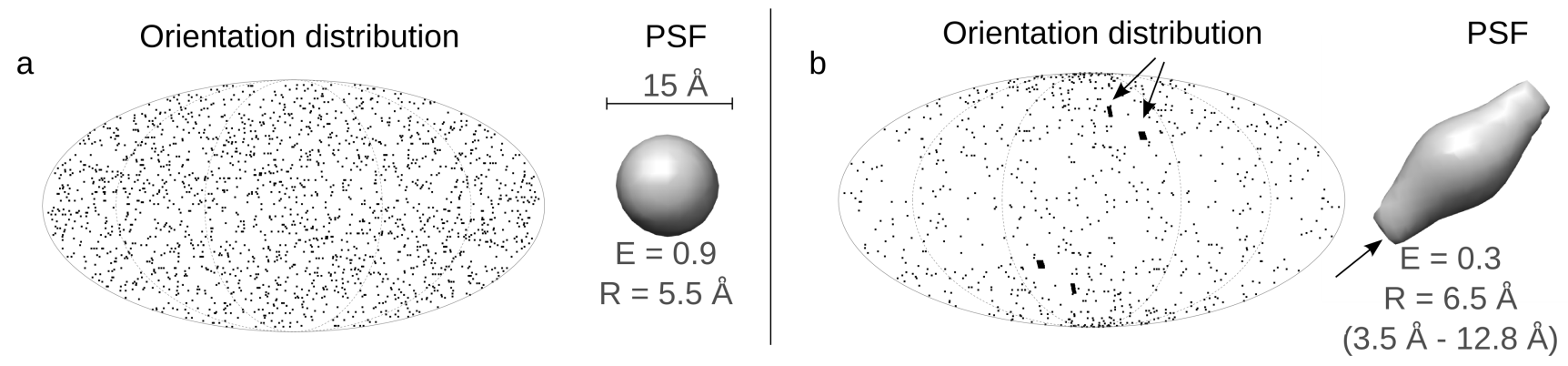

Figure 1. (a) A simulated uniform distribution of 1000 particle orientations provides uniform coverage of Fourier space and a spherical PSF with high efficiency (0.9). (b) A simulated distribution of 1000 particles with two strongly preferred orientations (arrows) fails to provide full Fourier space coverage and corresponds to a severely elongated PSF (arrow) with low efficiency (0.3).
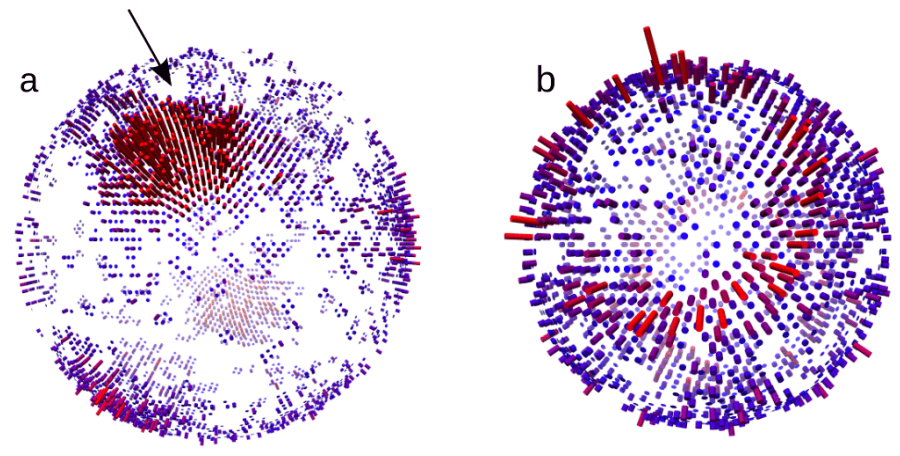

Figure 2. Collecting data at an optimal tilt angle can improve the efficiency of an orientation distribution with a strongly preferred orientation. (a) Orientation distribution of 1222 ribosome particles imaged imaged in ice with no additional support layer and without tilting. The particles show a strongly preferred orientation (arrow) and the efficiency of the distribution is 0.3. (b) Orientation distribution of 1222 ribosome particles imaged at optimal tilt angle of $29^{\circ}$ (recommended by the algorithm). The strongly preferred views are redistributed, providing better Fourier space coverage and improved efficiency (0.6). Improvement in both resolution and efficiency is achieved when these two datasets are combined. 